\title{
Effect of specific exercise strategy on need for surgery in patients with subacromial impingement syndrome: randomised controlled study
}

\author{
(C) $(1)(9)$ OPEN ACCESS
}

\author{
Theresa Holmgren PhD student ${ }^{1}$, Hanna Björnsson Hallgren PhD student ${ }^{2}$, Birgitta Öberg professor', \\ Lars Adolfsson professor ${ }^{2}$, Kajsa Johansson senior lecturer ${ }^{1}$
}

'Department of Medical and Health Sciences, Division of Physiotherapy, Linköping University, SE- 581 83, Linköping, Sweden; ${ }^{2}$ Department of Orthopaedics, University Hospital, SE-581 85, Linköping

\begin{abstract}
Objective To evaluate if a specific exercise strategy, targeting the rotator cuff and scapula stabilisers, improves shoulder function and pain more than unspecific exercises in patients with subacromial impingement syndrome, thereby decreasing the need for arthroscopic subacromial decompression.

Design Randomised, participant and single assessor blinded, controlled study.

Setting Department of orthopaedics in a Swedish university hospital.

Participants 102 patients with long standing (over six months) persistent subacromial impingement syndrome in whom earlier conservative treatment had failed, recruited through orthopaedic specialists.

Interventions The specific exercise strategy consisted of strengthening eccentric exercises for the rotator cuff and concentric/eccentric exercises for the scapula stabilisers in combination with manual mobilisation. The control exercise programme consisted of unspecific movement exercises for the neck and shoulder. Patients in both groups received five to six individual guided treatment sessions during 12 weeks. In between these supervised sessions the participants performed home exercises once or twice a day for 12 weeks.
\end{abstract}

Main outcome measures The primary outcome was the Constant-Murley shoulder assessment score evaluating shoulder function and pain. Secondary outcomes were patients' global impression of change because of treatment and decision regarding surgery.

Results Most $(97,95 \%)$ participants completed the 12 week study. There was a significantly greater improvement in the Constant-Murley score in the specific exercise group than in the control exercise group (24 points ( $95 \%$ confidence interval 19 to 28.0 ) $v 9$ points (5 to 13 ); mean difference between group: 15 points (8.5 to 20.6)). Significantly more patients in the specific exercise group reported successful outcome (defined as large improvement or recovered) in the patients' global assessment of change because of treatment: $69 \%$ (35/51) v24\% (11/46); odds ratio $7.6,3.1$ to $18.9 ; \mathrm{P}<0.001$. A significantly lower proportion of patients in the specific exercise group subsequently chose to undergo surgery: $20 \%$ (10/51) v63\% (29/46); odds ratio $7.7,3.1$ to $19.4 ; \mathrm{P}<0.001)$.

Conclusion A specific exercise strategy, focusing on strengthening eccentric exercises for the rotator cuff and concentric/eccentric exercises for the scapula stabilisers, is effective in reducing pain and improving shoulder function in patients with persistent subacromial impingement syndrome. By extension, this exercise strategy reduces the need for arthroscopic subacromial decompression within the three month timeframe used in the study.

Trial registration Clinical trials NCT01037673

\section{Introduction}

Subacromial impingement syndrome is said to have multiple causes. ${ }^{1}$ Several structures, such as the subacromial bursa, the tendons of the rotator cuff, the acromion, the coraco-acromial ligament, and the caput longum tendon of the biceps brachii muscle, are involved in the pathogenesis of subacromial impingement syndrome (fig $1 \Downarrow$ ). Multiple factors have been proposed as contributing to the development of subacromial impingement syndrome, including altered shoulder kinematics associated with dysfunction of the rotator cuff and scapular muscles, ${ }^{2-4}$ capsular tightness, ${ }^{5}$ poor posture,${ }^{67}$ and overuse secondary to sustained intensive work. ${ }^{89}$

Conservative treatment is the first choice, often with corticosteroid injection or different physiotherapy interventions, or both. ${ }^{10}$ Some studies have reported specific exercise treatment to be effective, ${ }^{11}{ }^{12}$ but the summarised evidence for its effectiveness is uncertain because of the limited number of studies and several with methodological concerns. ${ }^{13-15}$ Therefore 
more well designed and powered clinical trials evaluating specific exercise interventions are needed. When conservative treatment fails, arthroscopic subacromial decompression is recommended. ${ }^{16}{ }^{17}$ Surgery is performed in about $30 \%$ of all patients because conservative treatment fails. ${ }^{18}$ The number of patients who undergo arthroscopic subacromial decompression in Sweden has increased substantially in recent years, from 2287 in 2005 to 7959 in 2008 (Swedish Board of Health and Welfare, 2009). Studies have compared different exercise programmes with surgery and concluded that the two strategies have equivalent effects. A $65-80 \%$ success rate, improved shoulder function, and decreased pain have been reported after one year. ${ }^{12}{ }^{19} 20$ These results challenge the need for surgery. ${ }^{17}$ Some patients still do not respond to conservative and surgical intervention, indicating that the selection of patient for the different treatments is not optimal, and further research is required to identify those who really need surgery.

Despite limited evidence, several systematic reviews have suggested exercise treatment as the first line management for patients with subacromial impingement syndrome..$^{21-23}$ There is currently no consensus of the most appropriate exercise strategy. Further research is needed to develop evidence based exercise programmes that can be recommended and implemented for these patients. We evaluated whether a specific exercise strategy targeting the rotator cuff and scapula stabilisers improves shoulder function and pain more than unspecific exercises in patients with subacromial impingement syndrome, thereby decreasing the need for arthroscopic subacromial decompression.

\section{Methods}

\section{Participants and setting}

Patients aged 30-65 with a diagnosis of primary subacromial impingement syndrome from an orthopaedic specialist and on the waiting list for arthroscopic subacromial decompression were eligible for inclusion in the study. Participants were recruited from the department of orthopaedics, University Hospital, in Linköping, Sweden, between January 2008 and February 2010. The inclusion criteria were typical history with pain located in the proximal lateral aspect of the upper arm, especially with the arm raised; six month duration of the current episode; and lack of response to various conservative treatments (including exercise treatment) for at least three months. In addition, they had to have three of the following: impingement sign according to Neer, ${ }^{24}$ impingement sign according to Hawkins-Kennedy, ${ }^{25}$ positive result on Jobes test, ${ }^{26}$ and positive result on Patte's manoeuvre. ${ }^{27}$ Finally, the Neer's impingement test $^{24}$ (injection of $1 \mathrm{~mL}$ of $20 \mathrm{mg} / \mathrm{mL}$ triamcinolon mixed with $6 \mathrm{~mL}$ of $10 \mathrm{mg} / \mathrm{mL}$ mepivacain) was performed, and participants had to have a positive test result for inclusion in the study. The exclusion criteria were: radiologically verified malignancy; osteoarthritis of the glenohumeral joint; os acromiale decreasing the subacromial space; acromioclavicular arthritis; previous fractures in the shoulder complex or shoulder surgery on the affected side, or both; clinically verified polyarthritis, rheumatoid arthritis, fibromyalgia, instability in any joint of the shoulder complex, frozen shoulder, or symptoms from the cervical spine and pseudoparalysis; receipt of a corticosteroid injection in the previous three months for the current problem; or an inability to understand written and spoken Swedish. All patients provided written informed consent after being given oral and written information by the orthopaedic specialist at the inclusion visit.

\section{Procedures}

An independent physiotherapist prepared the random allocation sequence beforehand. Equal numbers of the two treatment alternatives, 55 of each, were prepared and concealed in opaque envelopes. These were then mixed by hand and numbered. At the inclusion visit, the orthopaedic specialist (HB) coded the patients consecutively. Treatment allocation was performed at the first visit to the physiotherapist, within two weeks of the inclusion visit. This procedure was used to secure blinding of the orthopaedic specialist acting as the assessor. The research physiotherapist received the envelope with the corresponding code revealing the assigned treatment alternative out of a central locked location just before the participants presented for one of the two treatments: specific exercises (specific exercise group) or unspecific exercises (control exercise group).

\section{Interventions \\ Both groups}

All patients received a subacromial corticosteroid injection at the inclusion visit, in line with usual practice. The exercises were introduced two weeks after the injection. Both groups received thorough information about their shoulder condition, ergonomical advice, and correction of their posture. The included patients visited the research physiotherapist (TH) once a week for the first two weeks and once every other week for the next 10 weeks (a total of seven visits). The first visit lasted about 60 minutes, and the subsequent visits lasted 30 minutes. In between these supervised sessions, participants performed home exercises once or twice a day for 12 weeks. An exercise diary was used in both groups to monitor adherence.

\section{Specific exercise group}

The programme was based on the latest scientific literature ${ }^{23} 2829$ and clinical experience and focused on strengthening eccentric exercises for the rotator cuff and strengthening concentric/eccentric exercises for the scapula stabilisers. The programme consisted of six different exercises: two eccentric exercises for the rotator cuff (supraspinatus, infraspinatus, and teres minor), three concentric/eccentric exercises for the scapula stabilisers (middle and lower trapezius, rhomboideus, and serratus anterior), and a posterior shoulder stretch. Each strengthening exercise was repeated 15 times in three sets twice daily for eight weeks. The posterior shoulder stretch was performed for 30-60 seconds and repeated three times twice daily. From week eight to week 12, the exercises were repeated once a day. The exercises were individually adjusted and progressed with increased external load by using weights and elastic rubber band at the physiotherapist visits once every other week during the whole rehabilitation period. When necessary, the physiotherapist performed manual treatment by stretching the posterior glenohumeral capsule and pectoralis minor during the visits. The pain monitoring mode ${ }^{30}$ was used to find the individual resistance for each patient. The patients were not allowed to exceed 5 on this $0-10$ scale when they performed the exercises; however, they were recommended to feel some pain during loading. After completion of an exercise session, increased pain had to revert to levels before exercise before the next session; otherwise, the external load was decreased. Great emphasis was placed on teaching good posture (thoracic spine extension and retracted shoulders) and to maintain this position during the exercises. After completion of the specific exercise programme (after 12 weeks), we recommended participants to maintain the daily home exercises for another two months. A 
more detailed description of the exercise programme can be found in the appendix on bmj.com.

\section{Control exercise group}

The control exercise programme consisted of six unspecific movement exercises for the neck and shoulder without any external load (shoulder abduction in the frontal plane, shoulder retraction, shoulder elevation, neck retraction, stretch of upper trapezius and pectoralis major. Each movement exercise was repeated 10 times, and each stretching exercise three times twice daily at home and once every other week at the physiotherapist visits. The patients did the same programme without any progression during the whole rehabilitation period. The unspecific exercise programme was thought to have a limited effect in patients with subacromial impingement syndrome and therefore acted as a control.

\section{Outcome measurements and assessment}

The same orthopaedic specialist evaluated all primary and secondary outcome measures at the inclusion visit before patients started the exercises (baseline) and after three months when patients had completed their exercise programme. The specialist was blinded to the group assignment throughout the study.

At the inclusion visit we documented age, sex, duration of subacromial pain, earlier treatment, drugs, occupation and duration of sick leave, and expectations of effects of treatment (completely restored, quite improved, not improved but some relief of the symptoms, or no expectations of being restored). Patients rated their work load on a four item scale dichotomised into two categories: light to moderate (not working with arms above the shoulders) and moderate to heavy (working with arms above the shoulders). We used the hospital anxiety and depression scale ${ }^{31}$ at baseline as a screening tool for mental distress. Ultrasonography was performed between inclusion and the three month assessment to investigate the status of the rotator cuff (intact, partial tear, or full thickness tear).

The primary outcome was the Constant-Murley shoulder assessment score. ${ }^{32}$ The score consists of both objective (range of motion and strength) and subjective measurements (pain assessment, work load, and leisure time activities), which are summarised in a score between 0 and 100. A higher score indicates better shoulder function. Secondary outcomes were measured with the disability of the arm, shoulder, and hand score, a questionnaire developed to measure upper extremity disability and symptoms. Patients' assessments are summarised by a score between 0 and 100 . A lower score indicates better shoulder function. ${ }^{33}$ We used a visual analogue scale (0-100 $\mathrm{mm}$ ) to measure the patient's perceived pain intensity at rest, during arm activity, and at night during the previous 24 hours at each assessment, ${ }^{34}$ and the EuroQol instrument (EQ-5D and EQ VAS) to evaluate health related quality of life (HRQL). ${ }^{35}$

At the three month assessment, after the end of treatment, the orthopaedic specialist asked the patient whether they still wanted or thought they needed surgery. The patient's global impression of change in symptoms because of treatment was also registered on a five point Likert scale: worse, unchanged, small improvement, large improvement, or recovered.

\section{Sample size calculation}

We calculated our sample size according to the Constant-Murley score. We estimated that we needed 82 patients to detect a mean 10 point difference between groups, ${ }^{19}{ }^{36}$ with a variability of 16 points ( $\beta=0.80$, two sided $\alpha=0.05$ ). To compensate for dropouts, we recruited an additional 20 patients.

\section{Data analysis}

We used one way analysis of variance for group differences at three month follow-up in primary and secondary outcomes using continuous scales, with adjustment for baseline levels of outcomes using group differences in mean change from baseline to three month follow-up as dependent variable.

The patient's global impression of change in symptoms because of treatment was dichotomised into large improvement (large improvement or recovered) or unimproved (slightly recovered, unchanged, or worse). We used Pearson's $\chi^{2}$ test to compare changes within and between groups, as well as the proportion of patients who still wanted or thought they needed surgery in the respective group at the three month assessment. We compared the percentage of successful outcomes between groups by calculating the odds ratios and their $95 \%$ confidence intervals with logistic regression. We used the statistical software package SPSS (version 18.0; SPSS, Chicago, IL) for all the analyses.

\section{Results}

A total of 152 patients were eligible for inclusion; 102 patients met the inclusion criteria and provided written informed consent to participate. Three weeks after inclusion, five patients were excluded: two patients developed a frozen shoulder, diagnosed by the physiotherapist three weeks after inclusion, and three patients changed their minds about participating in the study and declined participation at the first physiotherapist visit because of lack of time. A total of 97 patients were compliant with the study protocol from baseline to the three month assessment and were included in the statistical analysis (fig $2 \Downarrow$ ).

There were no differences in the background variables at baseline, except for more men in the specific exercise group (table $1 \Downarrow$ ), and no statistical differences between the groups in any of the outcome measures at baseline (table $2 \Downarrow$ ). Both groups had a low mean score on the hospital anxiety and depression scale, indicating limited mental distress (table $1 \Downarrow$ ). All patients included in the study expected a moderate or large treatment effect, with no significant differences between the two groups. The specific exercise group had significantly greater improvement than the control exercise group in the primary outcome (shoulder function and pain evaluated with Constant-Murley score), with a mean difference between groups of 15 points ( $95 \%$ confidence interval 8.5 to 20.6 ; table $2 \Downarrow$ ). The mean change in the Constant-Murley score was 24 points (19 to 28 ) in the specific exercise group and 9 points (5 to 13) in the control exercise group (table $2 \Downarrow$ ).

The mean change in the disabilities of the arm, shoulder, and hand score was significantly higher in the specific exercise group than the control exercise group, with a mean difference between groups of 8 points ( 2.3 to 13.7 ; table $2 \Downarrow$ ).

The mean change in visual analogue scores was significantly greater in the specific exercise group than in the control exercise group during the night, with a mean difference between groups of 20 points ( -30.9 to -7.2$)$ ). We found no significant differences in visual analogue scores between the groups for the mean change in pain during activity or at rest (table $2 \Downarrow$ ).

Health related quality of life, measured with the EQ-5D, was significantly higher $(\mathrm{P}<0.001)$ in the specific exercise group than in the control exercise group at the three month assessment (table $2 \Downarrow$ ). The mean change between the groups was 0.09 
( -0.07 to 0.18$)$. We identified no significant difference between the groups for the EQ-VAS $(\mathrm{P}=0.15)$.

Significantly more patients in the specific exercise group reported a successful outcome (defined as large improvement or recovered) according to the patients' global assessment of change because of treatment (69\% (35/51) v 24\% (11/46); odds ratio 7.6, 3.1 to $18.9 ; \mathrm{P}<0.001)$. A significantly lower proportion of patients in the specific exercise group subsequently chose surgery (20\% (10/51) v 63\% (29/46); odds ratio 7.7, 3.1 to 19.4; $\mathrm{P}<0.001)$.

\section{Adherence and co-interventions}

Each patient was scheduled to have six to seven visits to the physiotherapist during the 12 week exercise period. Most patients $(80 \%)$ in each group attended five or six visits. In the specific exercise group, 45 of the 51 patients completed their exercise diaries, and 44 of them missed fewer than 15 days of exercise out of 84 . Forty one patients out of the 46 in the control exercise group fulfilled their exercise diaries, and 40 of them missed fewer than 15 days of exercise out of 84 .

The number of patients using analgesics or anti-inflammatory drugs, or both, during the intervention period was similar in both control groups $(12 / 51(24 \%) v 15 / 46(33 \%))$.

\section{Discussion}

Patients with persistent subacromial impingement syndrome experience significantly greater improvements in shoulder function and pain after a specific exercise strategy over 12 weeks. In this randomised controlled study the specific exercise strategy, targeting the rotator cuff and scapula stabilisers, was compared with unspecific exercises. The exercise programme influenced the patients' choice about surgery as significantly more patients in the specific exercise group withdrew from the waiting list for surgery.

These positive results were achieved even though the participants had persistent symptoms that had not responded to at least three months of earlier conservative treatment (including exercise treatment) before inclusion.

\section{Content of the specific exercise strategy}

Several components in the specific exercise strategy are thought to contribute to these positive results. Firstly, the programme consisted of strengthening eccentric exercises for the rotator cuff and concentric/eccentric exercises for the scapula stabilisers in combination with manual mobilisation. Eccentric strength training has been successful in treating other tendonoses, especially those of the Achilles and patellar tendons. ${ }^{37-39}$ Why eccentric exercises are effective, however, is not fully understood. Eccentric exercises might provide a greater remodelling stimulus and also cause damage to the pathological nerves and neovessels around the affected tendon. ${ }^{40}$ Two recent pilot studies reported positive effects (decreased pain and increased shoulder function) after a 12 week period of eccentric exercises for the rotator cuff in patients with subacromial impingement syndrome. ${ }^{41}{ }^{42}$ Thus, eccentric exercises might be an important component of the rehabilitation strategy.

Strengthening exercises for the scapula stabilisers are thought to normalise the altered shoulder kinematics in patients with subacromial impingement syndrome $\mathrm{e}^{434}$ and, therefore, to reduce the risk for impingement. ${ }^{4}$ The muscles acting as scapula stabilisers make the scapula a stable basis from which the rotator cuff muscles can act. They also adjust the glenoid fossa in relation to the humeral head during arm movements. ${ }^{45} \mathrm{~A}$ combination of exercises for the rotator cuff and scapula stabilisers seems to be needed to minimise the risk for impingement and optimise rehabilitation for patients with subacromial impingement syndrome. In our current study, the exercises were combined with manual mobilisation when needed, which is supported by earlier reviews. ${ }^{13}{ }^{14}{ }^{21}$ With the current aim and design of this study, however, we were not able to evaluate the effect of each component in the strategy.

\section{Explanation of results}

Good adherence is required for a positive treatment effect. ${ }^{46}$ Because lack of time is a source of poor adherence, ${ }^{47}$ a small number of exercises is assumed to be beneficial for good adherence. Our programme consisted of only a few exercises, which could be completed in a reasonable time. In addition, regular follow-ups by the physiotherapist focused on hands-on guidance to facilitate appropriate performance of the exercises. Guided exercise treatment has been shown to be important, especially early in the rehabilitation phase when the patient needs support dealing with pain and disability. ${ }^{48}$ Pain during loading was allowed, as recommended by the pain monitoring model, ${ }^{30}$ and used to determine the progression of loading. The model was also used to support the patients in dealing with their experience of pain. This model has been successfully used in earlier studies that included patients with tendonopathy to ensure that the exercise treatment was well tolerated by the patients. ${ }^{30} 49$ It might even be a factor related to dose-response and important for efficacy.

The significant improvement in shoulder function seen in the specific exercise group was probably a response to the treatment. Little is known about natural recovery in patients with subacromial impingement syndrome and, as we did not include a third group with no treatment, we cannot evaluate the influence of natural recovery. The patients included in the present study, however, had persistent symptoms with low disability values at baseline, which has been reported to be associated with a poorer prognosis. ${ }^{50}$ Therefore, natural recovery is unlikely to explain the whole treatment effect, which is further supported by the differences between groups. The total treatment effect is also affected by the placebo effect. Attention, active treatment, ${ }^{51}$ and positive expectations ${ }^{52}$ are thought to contribute to the placebo effect. All patients were blinded to treatment assignment and had the same number of individual sessions with the physiotherapist. In addition, the expectations about treatment effects did not differ between the treatment groups and cannot explain the favourable results for the specific exercise group.

Furthermore, all patients received a corticosteroid injection, according to current practice and based on published evidence. ${ }^{53}{ }^{54}$ Though both groups reported significantly decreased pain over time, participants in the specific exercise group reported a significantly greater decrease; thus a positive effect of the corticosteroid injection cannot be the sole contributor to improvement.

The patient's own choice about surgery is thought to be a result of the positive treatment effect but should be interpreted with caution. Factors such as information given at the orthopaedic counselling might influence this choice. Therefore this information was standardised, emphasising that none of the existing treatments is superior.

\section{Comparison with other studies}

The specific exercise group experienced a large treatment effect, illustrated by a mean change of 24 points in the primary outcome (Constant-Murley score) after three months. These results are 
comparable with the results reported by several authors one to two years after arthroscopic subacromial decompression surgery. ${ }^{12}{ }^{36} 55$ Also the patients' global impression of change because of treatment reported by the specific exercise group (69\% much improved or recovered) is comparable with the success rates reported by the patients one year after arthroscopic subacromial decompression $(75 \%) .{ }^{56}$ The positive results of increased shoulder function and decreased pain are in line with earlier studies reporting a positive effect of exercise strategies. ${ }^{12}{ }^{57-59} \mathrm{~A}$ study by Bennell et al that used a strategy including manual treatment, however, reported no immediate effect after treatment. ${ }^{60}$ As some recent systematic reviews have expressed concerns over the methods of the earlier studies, no consensus exists regarding exercise treatment in patients with subacromial impingement syndrome. The main concerns have been assessor blinding, small sample sizes, and insufficient descriptions of the exercise programmes, including dose and adherence to the exercise protocol. ${ }^{13-15}$ Furthermore, studies evaluating the efficacy of exercise treatment in patients with persistent subacromial impingement syndrome who are on the waiting list for surgery are scarce. Two pilot studies evaluated eccentric exercises for the rotator cuff and reported favourable results. About $60-70 \%$ of the patients withdrew from the waiting list. ${ }^{41}{ }^{42}$ In the current study, an even higher percentage declined surgery ( $80 \%$ of the patients in the specific exercise group). Virta et al reported that only 10 of 97 patients needed surgery after 12 weeks of strengthening exercises. ${ }^{61}$ Their study design, however, lacked a control group. Dickens et al evaluated physiotherapy for patients on the waiting list for surgery, reporting that $26 \%$ of the patients in the intervention group withdrew from the waiting list, whereas all in the control group required surgery. ${ }^{57}$ Because their exercise programme was not standardised and the content, dose, and progression were not described in detail, however, the results cannot be compared with ours. Other studies have compared exercise treatment with surgery and reported similar positive outcomes after 12 months. ${ }^{12} 1920$ All these studies successfully used the supervised regimen by Bohmer et $\mathrm{al}^{62}$ and have contributed to the recommendation that exercises are used as first line management. The current study evaluating a new strategy for patients in whom earlier exercise treatments have failed adds valuable knowledge for managing patients with persistent subacromial impingement syndrome.

\section{Strength and limitations of the study}

The strengths of the present study are the strict study design, good attendance of the participants, and successful blinding. In addition, the standardised exercise protocol provides guidance about content, dose, and progression, which enables implementation into everyday practice. The study also has some limitations. Only one physiotherapist was involved in treatment and was not blinded to group assignment, which decreases the external validity of the results. The interaction with the physiotherapist was similar in both groups, however, and all patients received guidance and were encouraged to perform their home based exercises daily. The three month follow-up was a pragmatic choice, partly based on clinical experience that improvements with exercise programmes usually occur within that time, with an expected time to surgery of three months. All patients were recruited from the surgical waiting list of one orthopaedic clinic, however, which might affect the external validity of the results, though patients who were referred from primary care in the region of Östergötland (population 427106 , Central Bureau of Statistics 2009) to an orthopaedic specialist for surgery are thought to be representative of the studied population. Primary care patients with subacromial impingement syndrome have a better prognosis because of a better baseline outcome and shorter duration of pain ${ }^{50}$ than the studied population. Therefore, this specific exercise strategy might also be effective in this population. This positive treatment effect needs to be proved in a primary care setting with longer follow-up before possible implementation in primary care.

\section{Conclusion}

A specific exercise strategy, focusing on strengthening eccentric exercises for the rotator cuff and concentric/eccentric exercises for the scapula stabilisers, is effective in reducing pain and improving shoulder function in patients with persistent subacromial impingement syndrome. By extension, this exercise strategy reduces the need for arthroscopic subacromial decompression within the three month timeframe used in the study.

We acknowledge the support and contribution of the physiotherapy orthopaedic department of the University Hospital in Linköping, Sweden. We also thank Henrik Magnusson for all statistical assistance.

Contributors: TH, HB, BÖ, LA, and KJ conceived and designed the study protocol. $\mathrm{KJ}$ and BÖ procured the project funding. $\mathrm{TH}$ and $\mathrm{KJ}$ designed the physiotherapy interventions. TH did the statistical analyses with assistance from a statistician employed at the department of medical and health sciences. HB was the blinded assessor. TH drafted the manuscript, and BÖ, HB, LA and KJ contributed to the manuscript. All authors read and approved the final manuscript. $\mathrm{KJ}$ is guarantor.

Funding: This research received no specific grant from any funding agency in the public, commercial, or not-for-profit sectors. TH is funded in part by the research council in the south east of Sweden (FORSS).

\section{Competing interests: All authors have completed the ICMJE uniform} disclosure form at www.icmje.org/coi_disclosure.pdf (available on request from the corresponding author) and declare: no support from any organisation for the submitted work; no financial relationships with any organisations that might have an interest in the submitted work in the previous three years; no other relationships or activities that could appear to have influenced the submitted work.

Ethical approval: The study was approved by the ethics committee in Linköping (dnr: M124-07), and informed consent was given by all participants.

Data sharing: Physiotherapy protocols, statistical code, and dataset are available from the corresponding author.

1 Seitz AL, McClure PW, Finucane S, Boardman ND 3rd, Michener LA. Mechanisms of rotator cuff tendinopathy: intrinsic, extrinsic, or both? Clin Biomech (Bristol, Avon) 2011;26:1-12

2 Ludewig PM, Cook TM. Alterations in shoulder kinematics and associated muscle activity in people with symptoms of shoulder impingement. Phys Ther 2000;80:276-91.

3 Cools AM, Witvrouw EE, Mahieu NN, Danneels LA. Isokinetic scapular muscle performance in overhead athletes with and without impingement symptoms. J Athl Train 2005:40:104-10.

4 Kibler WB. Scapular involvement in impingement: signs and symptoms. Instr Course Lect 2006;55:35-43.

5 Tyler TF, Nicholas SJ, Roy T, Gleim GW. Quantification of posterior capsule tightness and motion loss in patients with shoulder impingement. Am J Sports Med 2000;28:668-73.

6 Lewis JS, Green A, Wright C. Subacromial impingement syndrome: the role of posture and muscle imbalance. J Shoulder Elbow Surg 2005;14:385-92.

7 Bullock MP, Foster NE, Wright CC. Shoulder impingement: the effect of sitting posture on shoulder pain and range of motion. Man Ther 2005;10:28-37.

8 Miranda H, Viikari-Juntura E, Martikainen R, Takala EP, Riihimaki H. A prospective study of work related factors and physical exercise as predictors of shoulder pain. Occup Environ Med 2001;58:528-34.

9 Frost P, Bonde JP, Mikkelsen S, Andersen JH, Fallentin N, Kaergaard A, et al. Risk of shoulder tendinitis in relation to shoulder loads in monotonous repetitive work. Am J Ind Med 2002;41:11-8.

10 Johansson K, Oberg B, Adolfsson L, Foldevi M. A combination of systematic review and clinicians' beliefs in interventions for subacromial pain. Br J Gen Pract 2002;52:145-52.

1 Brox JI, Gjengedal E, Uppheim G, Bohmer AS, Brevik JI, Ljungren AE, et al. Arthroscopic surgery versus supervised exercises in patients with rotator cuff disease (stage II impingement syndrome): a prospective, randomized, controlled study in 125 patients with a 2 1/2-year follow-up. J Shoulder Elbow Surg 1999;8:102-11. 


\section{What is already known in this topic}

Shoulder impingement syndrome is one of the most common shoulder conditions causing pain and loss of function

First choice of treatment is conservative, often with different exercise programmes; yet conclusive evidence to support the efficacy for

these programmes is lacking

\section{What this study adds}

This specific exercise strategy is effective in improving shoulder function and pain in patients with persistent subacromial impingement syndrome in whom earlier conservative treatment has failed. By extension, this could reduce the need for surgery within three months The standardised exercise protocol provides guidance about content, dose, and progression, which enables implementation into everyday practice

12 Haahr JP, Ostergaard S, Dalsgaard J, Norup K, Frost P, Lausen S, et al. Exercises versus arthroscopic decompression in patients with subacromial impingement: a randomised, controlled study in 90 cases with a one year follow up. Ann Rheum Dis 2005;64:760-4.

13 Green S, Buchbinder R, Hetrick S. Physiotherapy interventions for shoulder pain. Cochrane Database Syst Rev 2003;2:CD004258.

14 Kromer TO, Tautenhahn UG, de Bie RA, Staal JB, Bastiaenen CH. Effects of physiotherapy in patients with shoulder impingement syndrome: a systematic review of the literature. $J$ Rehabil Med 2009;41:870-80.

15 Kelly SM, Wrightson PA, Meads CA. Clinical outcomes of exercise in the management of subacromial impingement syndrome: a systematic review. Clin Rehabil 2010;24:99-109.

16 Ellman H. Arthroscopic subacromial decompression: analysis of one- to three-year results. Arthroscopy 1987;3:173-81.

17 Coghlan JA, Buchbinder R, Green S, Johnston RV, Bell SN. Surgery for rotator cuff disease. Cochrane Database Syst Rev 2008;1:CD005619.

18 Morrison DS, Frogameni AD, Woodworth P. Non-operative treatment of subacromial impingement syndrome. J Bone Joint Surg Am 1997;79:732-7.

19 Brox Jl, Staff PH, Ljunggren AE, Brevik Jl. Arthroscopic surgery compared with supervised exercises in patients with rotator cuff disease (stage II impingement syndrome). BMJ 1993:307:899-903.

20 Ketola S, Lehtinen J, Arnala I, Nissinen N, Westenius H, Sintonen H, et al. Does arthroscopic acromioplasty provide any additional value in the treatment of shoulder impingement syndrome? : a two-year randomised controlled trial. J Bone Joint Surg Br 2009;91:1326-34.

21 Michener LA, Walsworth MK, Burnet EN. Effectiveness of rehabilitation for patients with subacromial impingement syndrome: a systematic review. J Hand Ther 2004;17:152-64.

22 Dorrestijn O, Stevens M, Winters JC, van der Meer K, Diercks RL. Conservative or surgical treatment for subacromial impingement syndrome? A systematic review. J Shoulder Elbow Surg 2009;18:652-60.

23 Kuhn JE. Exercise in the treatment of rotator cuff impingement: a systematic review and a synthesized evidence-based rehabilitation protocol. J Shoulder Elbow Surg 2009;18:138-60.

24 Neer CS 2nd, Welsh RP. The shoulder in sports. Orthop Clin North Am 1977;8:583-91.

25 Hawkins RJ, Kennedy JC. Impingement syndrome in athletes. Am J Sports Med 1980;8:151-8.

26 Jobe FW, Jobe CM. Painful athletic injuries of the shoulder. Clin Orthop Relat Res 1983;1:117-24.

27 Leroux JL, Thomas E, Bonnel F, Blotman F. Diagnostic value of clinical tests for shoulder impingement syndrome. Rev Rhum Engl Ed 1995;62:423-8.

28 Cools AM, Dewitte V, Lanszweert F, Notebaert D, Roets A, Soetens B, et al. Rehabilitation of scapular muscle balance: which exercises to prescribe? Am J Sports Med 2007;35:1744-51.

29 Ellenbecker TS, Cools A. Rehabilitation of shoulder impingement syndrome and rotator cuff injuries: an evidence-based review. Br J Sports Med 2010;44:319-27.

30 Thomee R. A comprehensive treatment approach for patellofemoral pain syndrome in young women. Phys Ther 1997;77:1690-703.

31 Zigmond AS, Snaith RP. The hospital anxiety and depression scale. Acta Psychiatr Scand 1983;67:361-70

32 Constant CR, Murley AH. A clinical method of functional assessment of the shoulder. Clin Orthop Relat Res 1987;214:160-4.

33 Hudak PL, Amadio PC, Bombardier C. Development of an upper extremity outcome measure: the DASH (disabilities of the arm, shoulder and hand) [corrected]. The upper extremity collaborative group (UECG). Am J Ind Med 1996;29:602-8.

34 Carlsson AM. Assessment of chronic pain. I. Aspects of the reliability and validity of the visual analogue scale. Pain 1983:16:87-101.

35 Rabin R, de Charro F. EQ-5D: a measure of health status from the EuroQol group. Ann Med 2001;33:337-43.

36 Andersen NH, Sojbjerg JO, Johannsen HV, Sneppen O. Self-training versus physiotherapist-supervised rehabilitation of the shoulder in patients treated with arthroscopic subacromial decompression: a clinical randomized study. J Shoulder Elbow Surg 1999;8:99-101.

37 Magnussen RA, Dunn WR, Thomson AB. Nonoperative treatment of midportion achilles tendinopathy: a systematic review. Clin J Sport Med 2009;19:54-64

38 Kingma JJ, de Knikker R, Wittink HM, Takken T. Eccentric overload training in patients with chronic achilles tendinopathy: a systematic review. Br J Sports Med 2007;41:e3.

39 Jonsson $\mathrm{P}$, Alfredson $\mathrm{H}$. Superior results with eccentric compared to concentric quadriceps training in patients with jumper's knee: a prospective randomised study. Br J Sports Med 2005;39:847-50.

40 Rees JD, Wolman RL, Wilson A. Eccentric exercises; why do they work, what are the problems and how can we improve them? Br J Sports Med 2009;43:242-6.
41 Bernhardsson S, Klintberg $\mathrm{IH}$, Wendt GK. Evaluation of an exercise concept focusing on eccentric strength training of the rotator cuff for patients with subacromial impingement syndrome. Clin Rehabil 2011;25:69-78.

42 Jonsson $\mathrm{P}$, Wahlstrom $\mathrm{P}$, Ohberg L, Alfredson $\mathrm{H}$. Eccentric training in chronic painful impingement syndrome of the shoulder: results of a pilot study. Knee Surg Sports Traumatol Arthrosc 2006;14:76-81.

43 Cools AM, Witvrouw EE, Mahieu NN, Danneels LA. Isokinetic scapular muscle performance in overhead athletes with and without impingement symptoms. J AthI Train 2005;40:104-10.

44 McClure PW, Michener LA, Karduna AR. Shoulder function and 3-dimensional scapular kinematics in people with and without shoulder impingement syndrome. Phys Ther 2006;86:1075-90.

45 Cools AM, Witvrouw EE, Declercq GA, Danneels LA, Cambier DC. Scapular muscle recruitment patterns: trapezius muscle latency with and without impingement symptoms. Am J Sports Med 2003;31:542-9.

46 Deutscher D, Horn SD, Dickstein R, Hart DL, Smout RJ, Gurtvitz M, et al. Associations between treatment processes, patient characteristics, and outcomes in outpatient physical therapy practice. Arch Phys Med Rehabil 2009;90:1349-63.

47 Trost SG, Owen N, Bauman AE, Sallis JF, Brown W. Correlates of adults' participation in physical activity: Review and update. Med Sci Sports Exerc 2002;34:1996-2001.

48 Thorstensson CA, Roos EM, Petersson IF, Arvidsson B. How do middle-aged patients conceive exercise as a form of treatment for knee osteoarthritis? Disabil Rehabil . 2006;28:51-9

49 Silbernagel KG, Thomee R, Eriksson BI, Karlsson J. Continued sports activity, using a pain-monitoring model, during rehabilitation in patients with achilles tendinopathy: a randomized controlled study. Am J Sports Med 2007;35:897-906.

50 Thomas E, van der Windt DA, Hay EM, Smidt N, Dziedzic K, Bouter LM, et al. Two pragmatic trials of treatment for shoulder disorders in primary care: generalisability, course, and prognostic indicators. Ann Rheum Dis 2005;64:1056-61.

51 Krogsboll LT, Hrobjartsson A, Gotzsche PC. Spontaneous improvement in randomised clinical trials: meta-analysis of three-armed trials comparing no treatment, placebo and active intervention. BMC Med Res Methodol 2009;9:1.

52 Crow R, Gage H, Hampson S, Hart J, Kimber A, Thomas H. The role of expectancies in the placebo effect and their use in the delivery of health care: a systematic review. Health Technol Assess 1999;3:1-96.

53 Buchbinder R, Green S, Youd JM. Corticosteroid injections for shoulder pain. Cochrane Database Syst Rev 2003;1:CD004016.

54 Arroll B, Goodyear-Smith F. Corticosteroid injections for painful shoulder: a meta-analysis. Br J Gen Pract 2005;55:224-8.

55 Soyer J, Vaz S, Pries P, Clarac JP. The relationship between clinical outcomes and the amount of arthroscopic acromial resection. Arthroscopy 2003;19:34-9.

56 Patel VR, Singh D, Calvert PT, Bayley JI. Arthroscopic subacromial decompression: Results and factors affecting outcome. J Shoulder Elbow Surg 1999;8:231-7.

57 Dickens VA, Williams JL, Bhamra MS. Role of physiotherapy in the treatment of subacromial impingement syndrome: a prospective study. Physiotherapy 2005;91:159-64.

58 Camargo PR, Haik MN, Ludewig PM, Filho RB, Mattiello-Rosa SM, Salvini TF. Effects of strengthening and stretching exercises applied during working hours on pain and physical impairment in workers with subacromial impingement syndrome. Physiother Theory Pract 2009:25:463-75

59 Lombardi I,Jr, Magri AG, Fleury AM, Da Silva AC, Natour J. Progressive resistance training in patients with shoulder impingement syndrome: a randomized controlled trial. Arthritis Rheum 2008;59:615-22.

60 Bennell K, Wee E, Coburn S, Green S, Harris A, Staples M, et al. Efficacy of standardised manual therapy and home exercise programme for chronic rotator cuff disease: randomised placebo controlled trial. BMJ 2010;340:c2756.

61 Virta L, Mortensen M, Eriksson R, Möller M. How many patients with subacromial impingement syndrome recover with physiotherapy? A follow-up study of a supervised exercise programme. Adv Physiother 2009;11:166-73

62 Bohmer AS, Staff PH, Brox JI. Technical clinical note. Supervised exercises in relation to rotator cuff disease (impingement syndrome stages II and III): a treatment regimen and its rationale. Physiother Theory Pract 1998;14:93-105

Accepted: 25 November 2011

\section{Cite this as: BMJ 2012;344:e787}

This is an open-access article distributed under the terms of the Creative Commons Attribution Non-commercial License, which permits use, distribution, and reproduction in any medium, provided the original work is properly cited, the use is non commercial and is otherwise in compliance with the license. See: http://creativecommons.org/licenses/bync/2.0/ and http://creativecommons.org/licenses/by-nc/2.0/legalcode. 


\section{Tables}

Table 1 | Table 1 Background variables for two groups of patients with subacromial impingement syndrome according to randomised group. Values are numbers (percentages) unless stated otherwise

\begin{tabular}{lcc} 
& $\begin{array}{c}\text { Specific exercise group } \\
(\mathbf{n}=51)\end{array}$ & $\begin{array}{c}\text { Control exercise group } \\
(\mathbf{n}=46)\end{array}$ \\
Women & 14 & 22 \\
\hline Mean (SD) age (years) & $52(9)$ & $52(8)$ \\
\hline Median (range) duration of pain (months) & $24(6-120)$ & $22(48)$ \\
\hline Dominant side affected & $30(59)$ & $22 / 24$ \\
\hline Affected shoulder (right/left) & $32 / 18$ & $21(46)$ \\
\hline Occupation: & $22(43)$ & $25(55)$ \\
\hline Heavy load & $29(57)$ & $9(20)$ \\
\hline Light load & $9(18)$ & $34(74)$ \\
\hline On sick leave at start & & $6(13)$ \\
\hline Rotator cuff status*: & $33(65)$ & $6(13)$ \\
\hline Intact & $15(29)$ & $3.9(3.0)$ \\
\hline Partial tear & $3(6)$ & $2.5(2.6)$ \\
\hline Full thickness tear & $3.3(3.1)$ & \\
\hline HAD (0-21): & $2.2(2.3)$ & \\
\hline Mean (SD) anxiety & & \\
\hline Mean (SD) depression & & \\
\hline
\end{tabular}

$\mathrm{HAD}=$ hospital anxiety depression scale.

*Ultrasonographic examination. 
Table 2| Table 2 Mean (SD) of groups, mean change within groups ( $95 \%$ confidence interval), and mean differences ( $95 \%$ confidence interval) between groups adjusted for baseline scores

\begin{tabular}{|c|c|c|c|c|c|c|c|}
\hline \multirow[b]{2}{*}{ Groups* } & \multicolumn{2}{|c|}{ Baseline } & \multicolumn{2}{|c|}{ At 3 months } & \multicolumn{2}{|c|}{$\begin{array}{l}\text { Mean change within groups (baseline } \\
\text { to } 3 \text { months) }\end{array}$} & \multirow{2}{*}{$\begin{array}{l}\text { Mean differences } \\
\text { between groups } \\
\text { (baseline to } 3 \\
\text { months) }\end{array}$} \\
\hline & $\begin{array}{c}\text { Specific } \\
(n=51)\end{array}$ & Control $(n=46)$ & Specific $(n=51)$ & Control $(n=46)$ & Specific $(n=51)$ & Control $(n=46)$ & \\
\hline CM score & $48.5(15)$ & $43.5(15)$ & $72.5(19)$ & $52.5(23)$ & 24 (19 to 28 ) & $9(5$ to 13$))$ & 15 (8.5 to 20.6 ) \\
\hline DASH score & $30(14)$ & $35(19) \dagger$ & $16(15)$ & $29(19) \dagger$ & 14 (11 to 18$)$ & $6(2$ to 11$) \dagger$ & $8(2.3$ to 13.7$)$ \\
\hline EQ-5D & $0.67(0.23)$ & $0.62(0.23) \dagger$ & $0.82(0.14)$ & $0.69(0.24) \dagger$ & 0.16 (0.09 to 0.22$)$ & $0.07(-0.14$ to 0.01$)$ & $0.09(-0.07$ to 0.18$)$ \\
\hline EQ VAS & $68(15)$ & $62(20)$ & $75(20) \ddagger$ & $69(21) \ddagger$ & $6.6(0.4$ to 13$)$ & $6.1(-0.7$ to 13$) \ddagger$ & 0.5 (-8.7 to 9.8$)$ \\
\hline VAS rest & $15(19)$ & $20(21)$ & $10(14)$ & $20(25)$ & $4.1(-0.7$ to 11$)$ & -5 (-6.5 to 6.5$)$ & $-5.4(-14.1$ to 3.4$)$ \\
\hline VAS activity & $61(22)$ & $66(20)$ & $25(26)$ & $41(27)$ & 36 (27 to 46 ) & 25 (16 to 35$)$ & $-10.6(-23.6$ to 2.4$)$ \\
\hline VAS night & $46(28)$ & $40(30)$ & $15(22)$ & $27(27)$ & 32 (23 to 40 ) & $12(4$ to 21$)$ & $-20(-30.9$ to -7.2$)$ \\
\hline
\end{tabular}

*Groups: CM score=Constant-Murley shoulder assessment score 0-100 (100=maximum shoulder function); DASH score=disabilities of arm shoulder and hand score 0-100 ( $0=$ maximum shoulder function); EQ-5D index 1 to -0.59 ( $-0.59=$ lowest health related quality of life); EQ-VAS 0-100 ( $0=$ lowest health status); VAS=visual analogue scale $0-100$ ( $0=$ no pain).

$\dagger \mathrm{n}=44$.

$\ddagger \mathrm{n}=42$.

$\S \mathrm{n}=49$. 


\section{Figures}

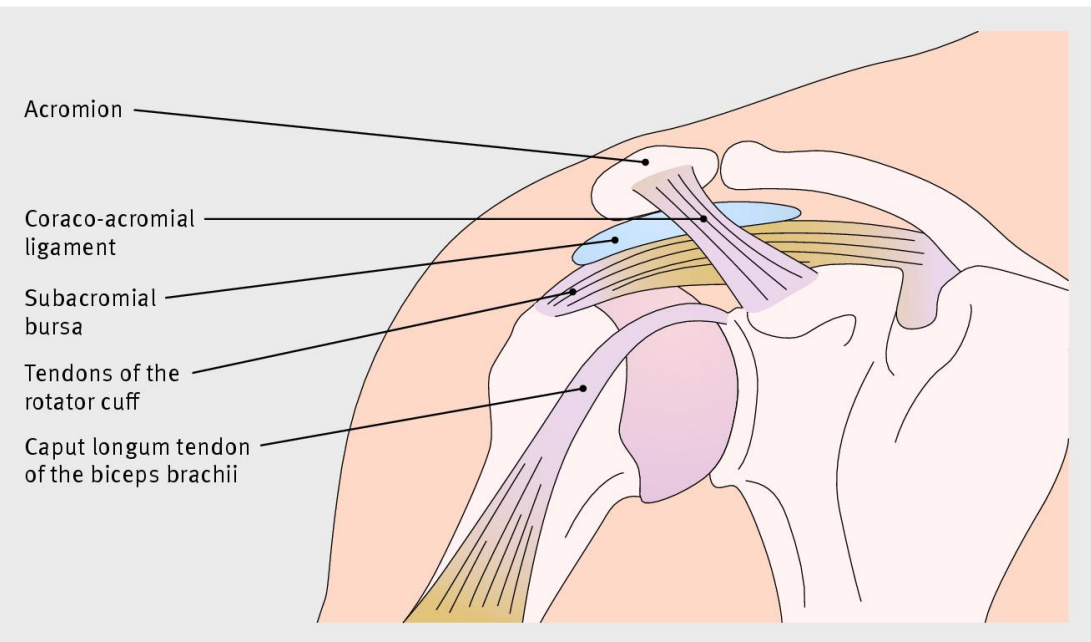

Fig 1 Anatomy of the shoulder

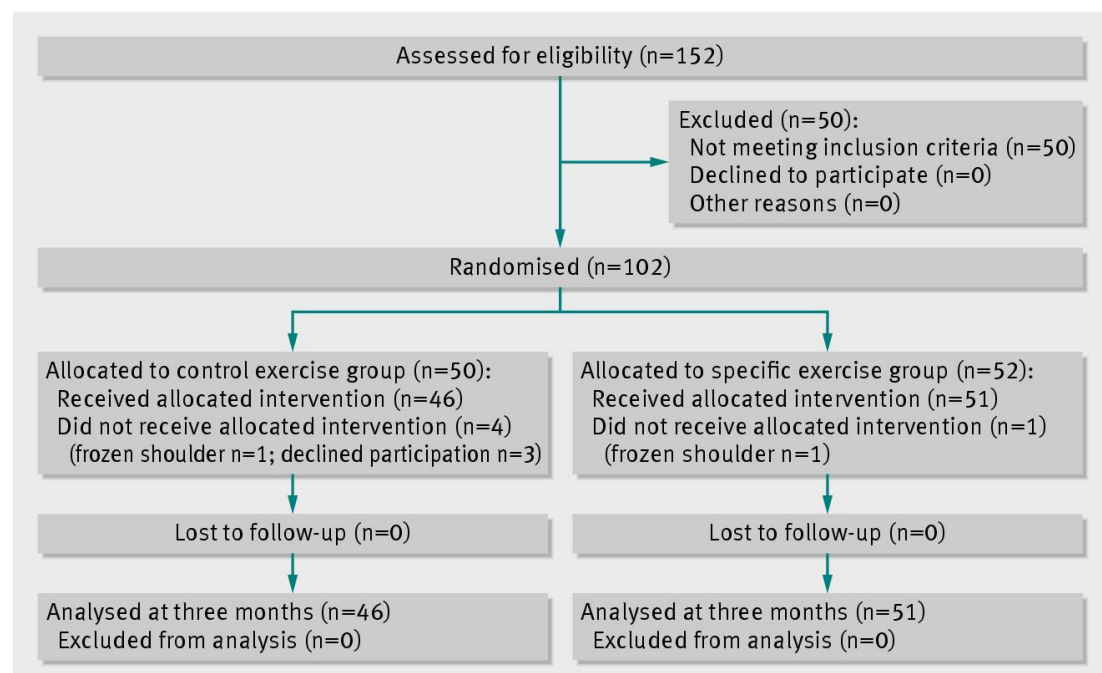

Fig 2 Flow of participants through study of effect of specific exercise strategy on patients with subacromial impingement syndrome 